\title{
A Comparative Model and Techno-Economic Analysis of Next Generation AON Ethernet and TDM PON
}

\author{
Kun Wang ${ }^{\mathrm{a}}$, Claus Popp Larsen ${ }^{\mathrm{a}}$, Anders Gavler ${ }^{\mathrm{a}}$, Bart Lannoo ${ }^{\mathrm{b}}$, Dominique Chiaroni ${ }^{\mathrm{c}}$, and \\ Mikhail Popova \\ (a) Acreo Netlab, Electrum 236, SE-164 40 Kista, Sweden, kun.wang@acreo.se, \\ (b) Ghent University - IBBT, Gaston Crommenlaan 8, B-9050 Gent, Belgium, \\ (c) Alcatel-Lucent Bell Labs, Route de Villejust, 91620 Nozay, France
}

\begin{abstract}
A global reference model covering next generation active and passive networks has been developed for techno-economic evaluations, and an extensive techno-economic analysis with a focus on CAPEX has been performed for 10G TDM PON and $1 \mathrm{G} \mathrm{AON} \mathrm{-} \mathrm{both} \mathrm{capable} \mathrm{of} \mathrm{delivering} \mathrm{1Gbit/s} \mathrm{to} \mathrm{end-users.} \mathrm{Two} \mathrm{major} \mathrm{cases} \mathrm{have} \mathrm{been} \mathrm{considered:} \mathrm{urban} \mathrm{and} \mathrm{rural}$ at green field deployment. The results show that AON is less expensive than PON solution in urban case while in rural case $10 \mathrm{G}$ TDM PON is more competitive.
\end{abstract}

Keywords: NGOA, AON, 10GPON, techno-economic

\section{INTRODUCTION}

Emerging new applications and network services, such as 3DTV, HDTV, triple-play, teleconferencing etc. will eventually increase bandwidth demands beyond $100 \mathrm{Mbps}$ per subscriber. Fiber to the home (FTTH) is a future proof infrastructure and is the only access technology that can readily support those new services, and next generation optical access should support 1 Gbps per subscriber.

There are basically two different kinds of FTTH architectures, namely active optical network (AON) and passive optical network (PON). AON is also known as active Ethernet or point-to-point (p2p) Ethernet or similar names, and there are different variants of it. AON is standardized in [1], [2], however without mentioning the term AON itself. PON - which is a point-to-multipoint ( $\mathrm{p} 2 \mathrm{mp}$ ) architecture - can be divided into time division multiplexing (TDM) PON and wavelength division multiplexing (WDM) PON. Current commercial TDM PON deployments are usually of the types GPON [1] or EPON [2]. Other types of PON have been proposed, and there are hybrids between TDM and WDM PONs and even between AON and PON. Within FSAN there are also major ongoing activities on next generation (NG) PON [3]. Today, AON in Europe represents $84 \%$ of total FTTH/B rollouts at end of 2009 [4]. Commercial deployments of AON offering 1 Gbps subscriber have begun with solutions from several vendors, e.g. [5], whereas PON offering 1 Gbps to multiple users is not yet available.

This paper focuses on a techno-economic study of NG optical access technologies at green field, specifically in capital expenditures (CAPEX). A simple model is proposed that allows for fair comparison of different access technologies. This model is then used on a scenario using 1 Gbps AON Ethernet and 10 Gbps TDM PON architectures. We believe this is the first techno-economic study between AON and PON offering 1 Gbps peak bandwidths to subscribers.

\section{A COMPARATIVE MODEL}

In order to fairly compare different technologies (e.g. with regards to techno-economics or energy efficiency) that are quite different in nature, one has to compare from a certain point of view. We assume a few comparative criteria:

- The goal of both technologies is to deliver IP based services to an end-user and the IP-packets will be transported and delivered over Ethernet.

- Bandwidth: A guaranteed average bandwidth rather than peak bandwidth in the network design is used as a benchmark for comparing different technologies. In this study, a sustainable $312 \mathrm{Mbps}$ (10Gbps divided by 1:32 split ratio) to every subscriber is applied in our network design; meanwhile the peak bandwidth is targeted for 
$1 \mathrm{Gbps} /$ subscriber for two reasons: a) AON system in this study is not able to beyond $1 \mathrm{Gbps}$ since only Gigabit optical interfaces were used towards the end-user in the AON network design; b) in order to avoid the high cost of CPEs ${ }^{1}$ with $10 \mathrm{Gbps}$ switch function, even if the 10G TDM PON has the possibility to occasionally reach 10 Gbps/subscriber with the help of dynamic bandwidth allocation, we still propose 1Gbps as a peak bandwidth in this paper.

- Central Office (CO) to home solution: The aggregation switch at CO site and the Customer Premises Equipment (CPE) switches at end-user site should be included for both AON and PON for a fair comparison. The CPE switch is here 1 Gbps capable ${ }^{1}$.

- Service area: both the AON and PON technologies are assumed to serve the same amount of end-users.

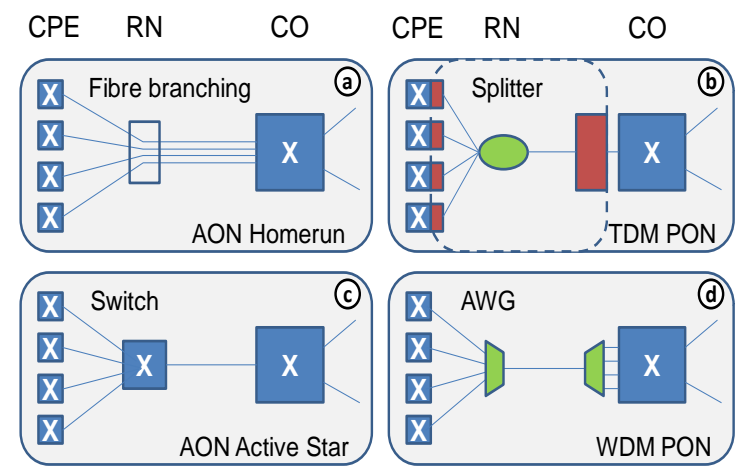

Fig. 1: The figure shows what equipment should be included in a comparative model. The abbrivations are; customer premises equipment (CPE), remote node (RN), central office (CO), and arrayed waveguide grating (AWG); Ethernet equipment is marked with blue, TDM equipment is red, and passive optical components are green.

To benchmark different FTTH options, we have identified a global network reference model [6] for the network in Fig. 1. This model points different network elements that need to be evaluated in the techno-economic analysis. The FTTH technologies envisaged are PON and AON in a way that enables a comprehensive study of these. WDM PON is included for comparison in the model but is not further studied here.

Fig. 1a shows an AON homerun architecture, in which a dedicated fiber connects each home to the CO, therefore it is also called point to point (P2P) Ethernet architecture. Fig. 1c is an AON star architecture, where many homes share one feeder fiber. In a star architecture, a remote node (RN) is deployed between the CO and the subscriber's premises. From network topology point of view the PON architecture is similar to AON star, but instead of putting an active switch, a passive splitter is used at the RN. The lines represent connections which in a techno-economic analysis should be translated to transceivers, fibers/cables, ducts and trenching. From $\mathrm{CO}$ to $\mathrm{RN}$ is the feeder part, and from RN till subscriber consist of distribution, drop and internal cabling. The AON active star and PON architectures have the same requirement on the amount of fibers (when both of them are using bi-directional transmission technology), whereas AON homerun need more fibers in the feeder part.

The blue aggregation node shown to the right in Fig. 1 is routinely included in the techno-economic models for AON and to some extend for WDM PON, but it is normally excluded in TDM PON studies since it is not part of the PON architecture itself. This fact is illustrated with the dashed line in Fig. 1b. However, the aggregation node is just as important for a complete TDM PON system as for the other architectures. If merely different TDM PONs were compared, the aggregation node could be neglected, but in a proper comparison between AON and PON it should be included to give a correct result.

\footnotetext{
${ }^{1} 1 \mathrm{Gbps}$ peak bandwidth leads to a standard CPE with 4 Gigabit Ethernet port. 10G TDM PON is able to offer peak 10Gbps, but the CPE need equip with $4 \times 10$ Gbps port and corresponding high speed switch fabric.
} 


\section{TECHNO-ECONOMIC ANALYSIS}

The total cost of the network is divided into two major parts: network elements and infrastructure. Network elements include: Ethernet switches, optical line terminal (OLT), optical network terminal (ONT), CPE, optical transceivers, and etc. The infrastructures part contains the cost of: remote node, splitter, fiber, ducts, civil work, fiber installation, trenching, and splicing etc.

In our detailed cost estimations we made a few assumptions:

- A minimum feeder distance (from $\mathrm{CO}$ to the remote node) of $2 \mathrm{~km}$ for the urban case (density 5000 inhabitants $/ \mathrm{km}^{2}$ ) and $10 \mathrm{~km}$ for the rural case (300 inhabitants $\left./ \mathrm{km}^{2}\right)$.

- A remote node is serving 1024 subscribers

- There are two types of fiber optical transmission solution: simplex fiber and duplex fiber solution. Simplex means only one fiber core is used for both signal transmitting and receiving, i.e. bi-directional (Bi-Di) transmission system. Duplex fiber system is of two separated fibers from which one of them is for transmitting and the other for receiving. In the access network area PON networks are commonly using Bi-Di transmission while AON networks can flexibly adopt both simplex and duplex fiber solutions. Since duplex fiber solution has been commonly used for many years in optical transmission systems e.g. SDH / SONET, Ethernet, etc., in this work we consider Bi-Di in a single fiber solution for PON network and duplex fiber cable solution for AON.

- We assume $10 \%$ more costs on fiber related infrastructure works (apart from fiber its own costs) for AON star than PON network due to the duplex fiber cable solution used in AON networks. By comparing Fig. 1(b) and (C), AON star has a similar topology as PON architecture. If Bi-Di transmission technology were used in both architectures, the amount of the fiber and corresponding civil works etc involved will be same. This add-on cost is more visible in the feeder part where truck cables are shared among thousands users, hence more duct space and splicing work etc is needed, however when the fiber cable is closer to the end-users the add-on costs of duplex cable is less.

- The AON homerun solution involves more fibers in the feeder part. Besides the fiber cost itself, the corresponding civil work costs are assumed 30\% more expensive than PON and AON active star solutions to incorporate the larger trench and ducts sizes.

- We considered the remote node costs for AON star is most expensive than others since the active equipment requires more robust location, power and cooling etc. remote node for AON homerun case cost lowest because it is only for fiber management purpose.

- For the network elements estimation, since $1 \mathrm{G}$ AON is commercially available, but $10 \mathrm{G}$ PON is not, two cost figures are given for the cost estimation of PON products in Fig. 2: a current estimated cost and a projected target cost. For the projected cost figures, we have assumed a distributed cost reduction to reach reasonable values in 2016. The price reduction assumptions have considered both mass-market factor and time factor. For general electronics part we assumed 10\% price reduction over every two years from 2010 till 2016, however for newly developed $10 \mathrm{G}$ PON optical components a dramatically decrease rate of $50 \%$ is used.

- For the cost of infrastructure and fiber related installation and civil work, we assume it will keep constant till 2016.

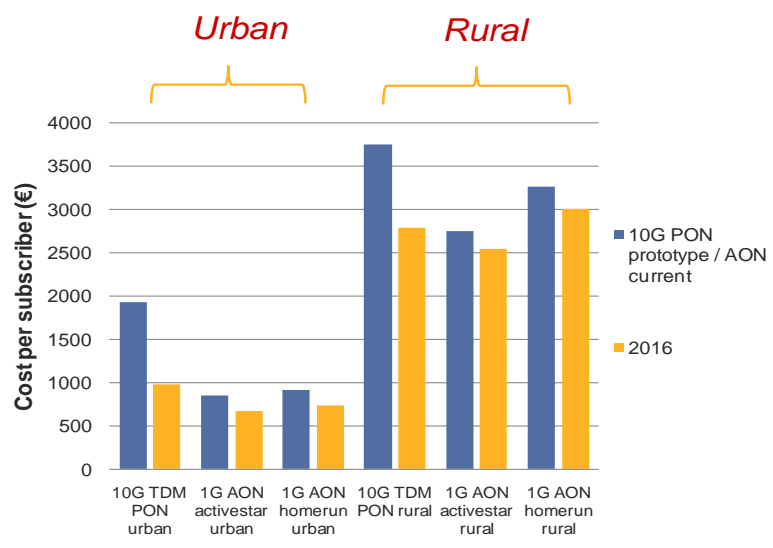

Fig. 2: Comparsion of curent and target cost per user

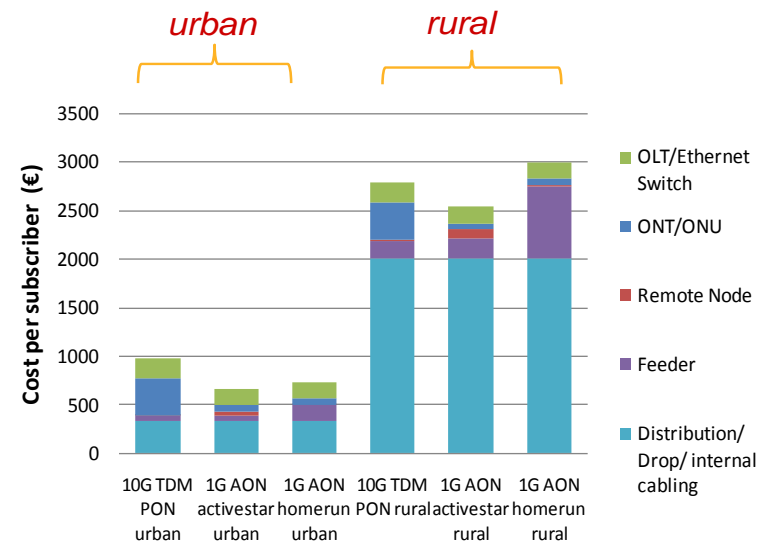

Fig. 3: CAPEX cost per subscriber of FTTH technologies 
Fig. 3 shows the cost breakdown for the considered technologies in 2016. It is clear that various aspects determine the cost for AON and PON technologies. AON active star is the most cost-effective solution in both urban and rural cases in terms of CAPEX. The OLT costs per subscriber are close among all architectures since it is shared cost among a group of users, however, the ONT cost is critical for $10 \mathrm{G}$ PON since $10 \mathrm{G}$ optics and electronics related to TDM (including DBA etc) have to be used at each end-user site. The most dominant cost factor is the infrastructure cost in feeder, distribution/drop/ internal cabling part across all access technologies; especially in the AON homerun case due to more fibers and larger civil works involved in the feeder part, and this cost enlarges when the technology is applied in the rural area.

We need to mention that the comparison is made between a 10G PONs technology and a $1 \mathrm{G}$ AON technology even if the comparison based on the same offered average bandwidth. The 10G PON is the only technology that has the possibility to offer a subscriber bit rate higher than $1 \mathrm{G}$, up to $10 \mathrm{G}$ in the extreme case. However, that would require a considerably more costly switch at the end-user site.

\section{CONCLUSION}

This techno-economic study has focused on green field deployments and on the CAPEX comparison between broadband access technologies: 10G TDM PON and $1 \mathrm{G}$ AON - both offering 1 Gbps peak bandwidth to end-users. For the urban case the two flavors of AON are expected to become cheaper than PON due to the high cost of $10 \mathrm{G}$ optics and electronics. For the rural case, PON is more competitive. However, the majority of the cost in all cases arises from trenching and civil works.

Observe that OPEX, which was not included here, regulation, business models, and other factors may have higher impact on a technology choice than CAPEX. Also trenching costs in especially the urban case may vary considerably depending on different parameters, but that will affect the technologies with the same factor.

\section{ACKNOWLEDGEMENT}

The research leading to these results has received funding from the European Community's Seventh Framework Programme (FP7) under project ALPHA n 212352 and OASE n 249025.

\section{REFERENCES}

[1] ITU-T Recommendation G.986, 1 Gbit/s point-to-point Ethernet based optical access system

[2] IEEE 802.3ah Ethernet in the First Mile Task Force: http://www.ieee802.org/3/ah

[3] Full service access network, FSAN: www.fsanweb.com

[4] IDATE, European FTTH/B market at end 2009, http://www.idate.org

[5] Ericsson Gigabit point-to-point access portfolio, EFN432, http://www.ericsson.com/news/ 100318_gigabit_p2p_20100510174827

[6] C.P. Larsen et al, "Comparison of Active and Passive Optical Access Networks", CTTE 2010, Ghent, Belgium, June 2010 